\title{
Evaluación de dietas para alevines de arawanas (Osteoglossum bicirrhosum) en el piedemonte amazónico de Colombia
}

\author{
M.Trujillo, D.M.; Rojas, A.M.; Murcia, B.; Chaves, L.C.; Pimentel, G.A. \\ Grupo de Investigación en Biodiversidad y Desarrollo Amazónico "BYDA", \\ Universidad de la Amazonia, Calle 17 Diagonal 17 con Carrera 3F, Barrio Porvenir Florencia-Caquetá, \\ Colombia, Tel: 3184019771. E-mail: 1.chaves@udla.edu.co
}

\begin{abstract}
Resumen
M.Trujillo, D.M.; Rojas, A.M.; Murcia, B.; Chaves, L.C.; Pimentel, G.A.: Evaluación de dietas para alevines de arawanas (Osteoglossum bicirrhosum) en el piedemonte amazónico de Colombia. Rev. vet. 28: 2, 145-151, 2017. El objetivo del trabajo fue comparar una dieta tradicional (concentrado proteico $45 \%$ de proteínas, TC) versus dos alimentos vivos: Poecilia reticulata (T1: guppys: $27 \%$ proteínas) y Coptotermes formosanus (T2: termitas: $22 \%$ proteínas), con el fin de desarrollar estrategias que permitan el óptimo desarrollo de alevines de arawana (O. bicirrhosum). Ejemplares de $O$. bicirrhosum $(\mathrm{n}=54)$ en etapa VII fueron estudiados en un sistema SCR (recirculación cerrada de agua) que incluyó 9 acuarios ( 6 individuos por acuario, tres réplicas cada uno). El grupo T1 presentó los mejores resultados para las 3 réplicas, con ganancia de talla: $0,11 \mathrm{~mm} /$ día, peso: 0,230 g/día, e índice de supervivencia: $100 \%$. En segundo lugar, el grupo TC obtuvo ganancia de peso: 0,169 g/día. En tercer término, el grupo T2 logró ganancia de peso: $0,084 \mathrm{~g}$ /día e índice de supervivencia: $98 \%$. Se concluye resaltando que no son necesarios altos porcentajes de proteínas $(45 \%)$ para obtener mayor peso y que la mejor dieta alternativa fue el alimento vivo a base de guppys, al lograr el mejor desarrollo de los alevines.
\end{abstract}

Palabras clave: pez arawana, alevines, alimento, proteínas, peso, talla, supervivencia.

\begin{abstract}
M.Trujillo, D.M.; Rojas, A.M.; Murcia, B.; Chaves, L.C.; Pimentel, G.A.: Evaluation of diets for arawana fingerlings (Osteoglossum bicirrhosum) in the amazon foothills of Colombia. Rev. vet. 28: 2, 145-151, 2017. The objective of the study was to compare a traditional diet (protein concentrate $45 \%$, TC) versus two live foods: Poecilia reticulata (T1: guppys: $27 \%$ proteins) and Coptotermes formosanus (T2: termites: $22 \%$ proteins), to develop strategies that all ow the optimal development of arawana fingerlings (O. bicirrhosum) phase VII. The specimens $(\mathrm{n}=54)$ were studied in an SCR system (closed water recirculation) that included 9 aquariums (6 individuals per aquarium, three replicates each). The T1 group presented the best results for the 3 replicates, with gain of size: $0.11 \mathrm{~mm} / \mathrm{day}$, weight: $0.230 \mathrm{~g} / \mathrm{day}$, and survival rate: $100 \%$. Second, the TC group had a weight gain of $0.169 \mathrm{~g} /$ day. Third, the T2 group achieved weight gain: $0.084 \mathrm{~g}$ /day and survival rate: $98 \%$. It is concluded that high protein percentages (45\%) are not necessary to obtain greater weight and that the best alternative diet in achieving the best development of fingerlings was the live food based on guppys.
\end{abstract}

Key words: fish arawana, alevines, food, proteins, liveweight, size, survival.

\section{INTRODUCCIÓN}

En Colombia, especialmente en las cuencas de los ríos Amazonas y Orinoco, las cuales se caracterizan por su gran potencial hidrobiológico ${ }^{17}$, se encuentra distribuida una gran variedad de especies de peces que pueden presentar un considerable potencial acuícola, entre ellas las del genero Osteoglossum (Osteoglossiformes: Osteoglossidae) conocidas como arawanas ${ }^{1}$.

Las arawanas son especies ornamentales exóticas, cuya coloración y movimiento armónico las vuelve lla-

Recibido: 5 octubre 2016 / Aceptado: 18 mayo 2017 mativas en la acuariofilia ${ }^{14}$, siendo consideradas como uno de los recursos ícticos más promisorio de la amazonia por su alto valor comercial ${ }^{12}$. Sin embargo, la gran mayoría de los ejemplares para este fin, son obtenidos del medio natural en pesca indiscriminada, lo que los ha tornado vulnerables ${ }^{22}$.

Esta situación se puede minimizar a través de la producción ex situ, lo que requiere condiciones esenciales para su manejo, como la calidad del agua y la nutrición ${ }^{8}$. Para garantizar que la aptitud del agua sea suficiente y adecuada para el mantenimiento de los organismos acuáticos, se puede implementar el sistema de recirculación cerrada (SCR), donde el agua es some- 
tida a una serie de tratamientos que incluye la remoción de sólidos y de compuestos nitrogenados, así como la oxigenación del sistema, permitiendo reutilizarla y controlar su calidad ${ }^{29}$.

Otro de los factores de gran importancia en la producción ex situ, es el alimento suministrado. A nivel nutricional, las proteínas son uno de los componentes más importantes que afectan el rendimiento piscícola, y también uno de los más $\operatorname{costosos}^{13}$. Por su parte, los macronutrientes son indispensables en la dieta porque aportan la energía necesaria para la supervivencia de los peces ${ }^{11}$.

Estas condiciones son las que determinan el éxito y la rentabilidad del sistema productivo, ya que la eficiencia con la que el pez transforma los nutrientes suministrados en músculo, lo puede llevar a desarrollar estrategias alimenticias que permitan disminuir los costos por alimento, incrementar su masa muscular y mantener el bienestar del animal ${ }^{27}$.

En esta investigación se planificó comparar una dieta tradicional (concentrado proteico $45 \%$ de proteínas) versus dos alimentos vivos: Poecilia reticulata (guppys: $27 \%$ proteínas) y Coptotermes formosanus (termitas: 22\% proteínas), con el fin de desarrollar estrategias que permitan el óptimo desarrollo de alevines de arawana (Osteoglossum bicirrhosum) en etapa VII.

\section{MATERIAL Y MÉTODOS}

Lugar de estudio. La investigación se desarrolló en el Laboratorio de Investigación de Ictiología, Universidad de la Amazonia (Florencia-Caquetá, Colombia), entre las coordenadas geográficas $1^{\circ} 36^{\prime} 28.83^{\prime \prime} \mathrm{N}, 75^{\circ} 36^{\prime} 23.22^{\prime \prime} \mathrm{S}$, a una altitud promedio de $377 \mathrm{msnm}$, humedad relativa entre 80 y $85 \%$, temperatura ambiente promedio de $28^{\circ} \mathrm{C}$, precipitación promedio anual de $3.835 \mathrm{~mm}$ y brillo solar aproximado de 2000 horas.

Unidades experimentales. Se dispuso de 60 alevinos de arawanas (O. bicirrhosum) de la misma cama$\mathrm{da}$, con peso promedio de 2,65 $\pm 0,39$ g, longitud de $8,28 \pm 0,40 \mathrm{~cm}$ y edad aproximada de 10 días. Los ejemplares se encontraban en la fase VII de desarrollo según el establecimiento de producción acuícola de la zona). Trasladados al laboratorio se mantuvieron 15 días en cuarentena, en acuarios con termostato a temperatura de 26 a $28^{\circ} \mathrm{C}$ y pH de $6,7^{17}$.

Análisis del alimento. Antes de iniciar los tratamiento se verificó el contenido proteico de cada una de las dietas alternativas, a saber: peces Poecilia reticulata (guppy o lebistes, tratamiento T1) e insectos Coptotercha).

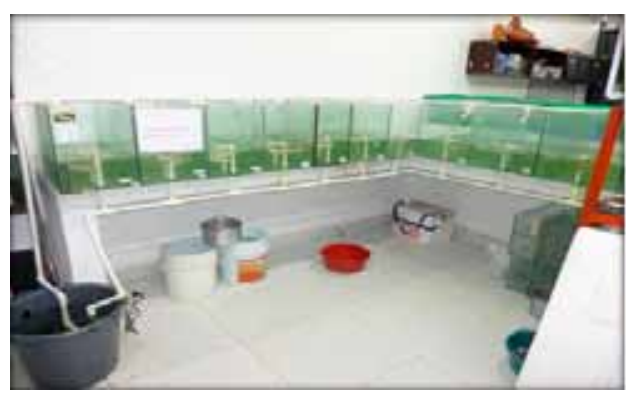

mes formosanus (termitas o comejenes, tratamiento T2). El análisis se realizó mediante los métodos Kjeldahl y micro-Kjeldahl y se confirmaron en el Laboratorio de Biotecnología de la Universidad de San Buenaventura (Cali, Colombia).

Diseño experimental. Luego de una fase de cuarentena, se planteó un diseño experimental multifactorial con 54 alevinos de $O$. bicirrhosum distribuidos completamente al azar en tres tratamientos (TC, T1, T2) con dos repeticiones cada uno (R1, R2) por un periodo de 78 días (Tabla 1). Se utilizó una metodología adaptada de la Universidad Michoacana (México) ${ }^{19}$, donde los nueve acuarios (de $60 \times 30 \mathrm{~cm}$ ), estuvieron conectados mediante un SCR, con 3 termostatos marca Resun de $200 \mathrm{w}$, temperatura constante de $28^{\circ} \mathrm{C}$ y volumen total de 295 litros de agua.

El SCR (Figura 1) consistió en un recipiente plástico con capacidad de 100 litros (recepción del agua recirculada), una bomba de inmersión de 800 litros/h conectada con tubería de PVC de $1 / 2$ " y llaves terminales de 1/2" (salida). El agua se obtuvo como "agua madura" con tratamiento de anticloro del acueducto municipal. El mantenimiento de la sanidad y la prevención de la descomposición del agua se hizo mediante recambios

Figura 1. Sistema de recirculación cerrada (SCR) utilizado en el estudio.
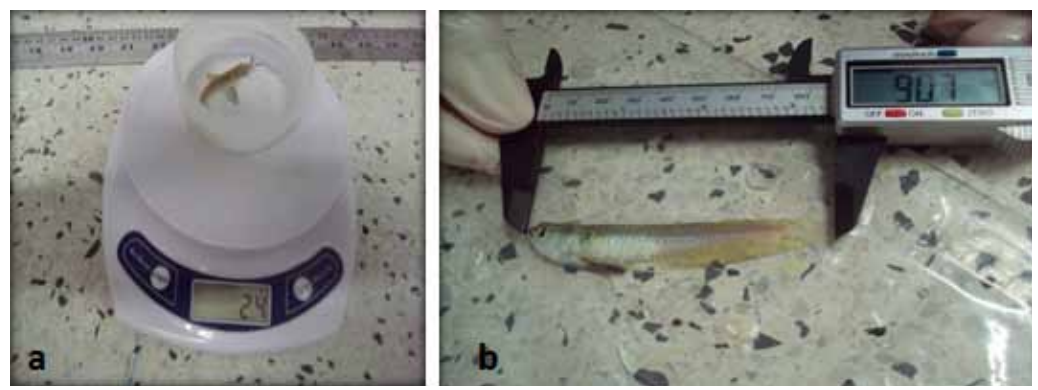

Figura 2. Mediciones biométricas de peso (izquierda) y longitud (dere- 
diarios (sifoneo) del 10\% y cada 8 días del 40 al 50\%, registrándose diariamente el $\mathrm{pH}^{25}$.

El suministro del alimento se realizó de acuerdo a la metodología que tiene en cuenta la biomasa registrada en cada muestreo ${ }^{14}$, iniciando con 9,54 g/tratamiento. La ración diaria se dividió en tres comidas y se suministró en el mismo horario (7:00 am, 1:30 pm y 6:00 pm) durante el estudio.

Determinación de parámetros productivos. A lo largo de la investigación se realizaron muestreos quincenales que abarcaron el $100 \%$ de la población de cada tratamiento. Se registró el peso $(\mathrm{g})$ mediante balanza digital y la longitud total $(\mathrm{cm})$ con calibrador digital (Figura 2). Las variables calculadas fueron: ganancia de peso [peso final $(\mathrm{g})$ - peso inicial $(\mathrm{g})$ ], ganancia de talla [talla final $(\mathrm{cm})$ - talla inicial $(\mathrm{cm})$ ], ganancia diaria de peso [GDP g/día = peso final - peso inicial $/$ días del ciclo de producción], tasa de crecimiento específico $[\mathrm{TCE}=$ peso final - peso inicial / tiempo de estudio * 100], y tasa de sobrevivencia [ $\mathrm{N}^{\mathrm{o}}$ final de peces $/ \mathrm{N}^{\mathrm{o}}$ inicial de peces) $* 100^{5,14,23}$.

Sistematización de las ganancias de peso, supervivencia y crecimiento. Estos parámetros fueron obtenidos a partir de cálculos propuestos por expertos en dichos temas ${ }^{21,24}$, a saber:

A. Tasa de supervivencia:

1) el peso promedio ganado por día se calculó en base a la expresión:

$$
P g=\frac{W_{f}-W_{i}}{t}
$$

donde $P g=$ peso ganado por día (GDP), $W_{i}=$ peso inicial, $W_{f}=$ peso final y $t=$ tiempo en (días).

2) porcentaje de peso ganado:

$$
P g(\%)=100 \frac{(\text { peso final }- \text { peso inicial) }}{\text { peso inicial }}
$$

3) tasa de supervivencia (TS):

$$
T S=\frac{\text { número final de organismos }}{\text { número inicial de organismos }} \times 100
$$

B. Tasa de crecimiento:

Las ganancias de peso (GP) fueron obtenidas a partir de la siguiente expresión:

$$
G P=\text { peso promedio final }(\mathrm{g}) \text { - peso promedio inicial }(\mathrm{g})
$$

Las tasas de crecimiento específico (TCE) se calcularon con la formula ${ }^{2}$ :

$$
T C E=100 \times[\text { Ln peso final }(\mathrm{g})-\text { Ln peso inicial }(\mathrm{g})] / t
$$

La expresión “(t)” corresponde al número de días.

Para la orientación de la tasa de crecimiento absoluto (TCA) se utilizó la fórmula ${ }^{4}$ :

$$
\begin{gathered}
T C A=[\text { peso final }(\mathrm{g})-\text { peso inicial }(\mathrm{g})] /(T 2-T 1) \\
\text { TCA }=\text { Tasa de crecimiento absoluto } \\
T 2=\text { tiempo en duración en dias de cultivo }
\end{gathered}
$$

$$
T 1 \text { = tiempo de iniciación del cultivo }
$$

El factor de condición $(K)$, fue calculado a partir de la relación matemática entre el peso y la longitud ${ }^{6}$ :

$$
\begin{gathered}
K=\frac{P}{L 3} \times 100 \\
P=\text { peso promedio }(\mathrm{gr}) \\
L=\text { longitud promedio }(\mathrm{cm})
\end{gathered}
$$

Estadísticas. Los resultados se analizaron con el programa estadístico Statgraphics $5.1 \AA$, mediante un ANOVA con nivel de significancia de 0,05. Las diferencias estadísticas se estimaron por el test de Tuckey.

Manejo ético. Se tuvo en cuenta que las condiciones de mantenimiento de los animales fueran adecuadas y que el número de ejemplares utilizados no excediera el rango necesario, evitando todo sufrimiento físico inútil. Para la iniciación de la investigación fue necesario realizar la solicitud del permiso de bioética a la autoridad universitaria, el cual fue oportunamente otorgado.

\section{RESULTADOS}

El método de Kjeldahl indicó el contenido de proteínas del alimento concentrado (45\%), de los guppys (P. reticulata, 27\%) y de las termitas (C. formosanus, $22 \%$ ). El análisis de la calidad del agua (SCR) arrojó un $\mathrm{pH}$ de 6,3 (Figura 3) y una temperatura promedio de $28^{\circ} \mathrm{C}$ (Figura 4).

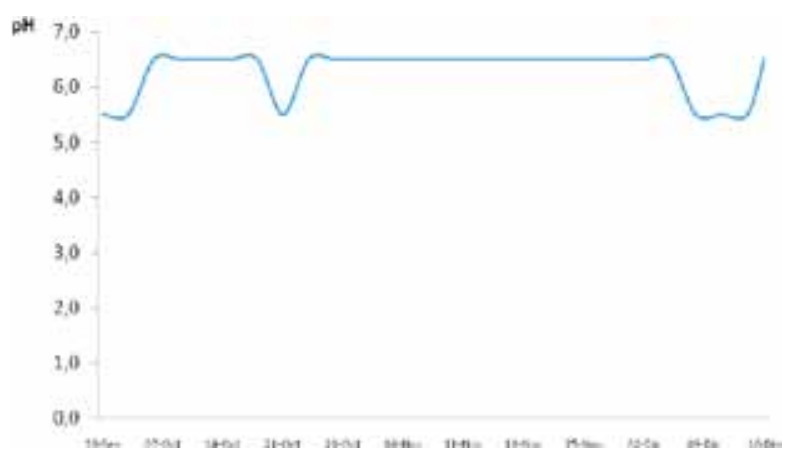

Figura 3. Valores de $\mathrm{pH}$ en el agua (SCR) durante el cultivo de alevines de arawanas.

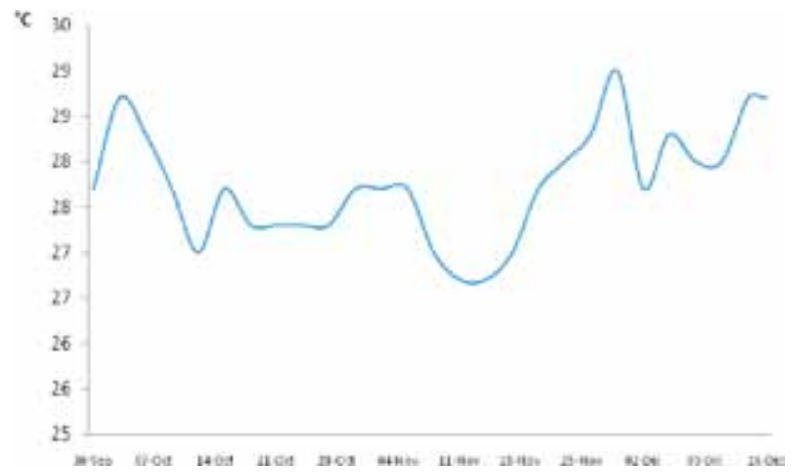

Figura 4. Temperaturas registradas en el SCR (sistema de recirculación de agua). 
Tabla 2. Promedios de supervivencia y aumentos de peso y longitud en alevines según tratamiento.

\begin{tabular}{lccc}
\hline resultados & $\mathrm{TC}$ & $\mathrm{T} 1$ & $\mathrm{~T} 2$ \\
\hline supervivencia $(\%)$ & 100 & 100 & 98 \\
peso inicial $(\mathrm{g})$ & 2,44 & 2,86 & 2,65 \\
peso final $(\mathrm{g})$ & 15,66 & 20,83 & 9,17 \\
ganancia diaria $(\mathrm{g})$ & 0,169 & 0,230 & 0,084 \\
peso ganado $(\%)$ & 54,18 & 62,83 & 24,60 \\
longitud inicial $(\mathrm{cm})$ & 8,15 & 8,25 & 8,42 \\
longitud final $(\mathrm{cm})$ & 15,08 & 16,58 & 12,75 \\
\hline
\end{tabular}

TC: concentrado $45 \%$, T1: guppys $27 \%$, T2: termitas $22 \%$.

Tabla 3. Ganancia de peso, biomasa y crecimiento según el alimento consumido.

\begin{tabular}{cccccccccc}
\hline A & R & BTI & BTF & GDP & TCE & TCA & Ki & Kf & SV \\
\hline TC & R1 & 2.33 & 17.13 & 14.8 & 2.56 & 0.255 & 0.0051 & 0.00452 & 100 \\
TC & R2 & 2.47 & 13.67 & 11.2 & 2.19 & 0.193 & 0,00479 & 0,00428 & 100 \\
TC & R3 & 2.52 & 16.18 & 13.6 & 2.38 & 0.235 & 0,00391 & 0,00475 & 100 \\
T1 & R4 & 2.68 & 21.28 & 18.6 & 2.66 & 0.320 & 0,00473 & 0,00525 & 100 \\
T1 & R5 & 2.67 & 19.75 & 17.0 & 2.56 & 0.294 & 0,00445 & 0,00489 & 100 \\
T1 & R6 & 3.22 & 21.47 & 18.2 & 2.43 & 0.314 & 0,00452 & 0,0051 & 100 \\
T2 & R7 & 2.50 & 8.87 & 6.37 & 1.62 & 0.109 & 0,00232 & 0,00432 & 100 \\
T2 & R8 & 2.52 & 10.02 & 7.50 & 1.76 & 0.129 & 0,00252 & 0,00416 & 100 \\
T2 & R9 & 2.92 & 8.62 & 5.70 & 1.38 & 0.098 & 0,00445 & 0,00477 & 98 \\
\hline
\end{tabular}

A: alimentos (TC: concentrado, T1: guppys, T2: termitas), R (réplicas), BTI (biomasa total inicial, gramos), BTF (biomasa total final, gramos), GDP (ganancia de peso individual, gramos), TCE (tasa de crecimiento especifico, \% día), TCA (tasa de crecimiento absoluto, g/día), Ki (factor de condición inicial), Kf (factor de condición final), SV (sobrevivencia para cada réplica, en \%).

Las 3 réplicas de cada uno de los 3 tratamientos se identifican de la siguiente manera TC (concentrado: R1, R2 y R3), T1 (guppys: R4, R5, R6) y T2 (termitas: R7, R8 y R9), como indica la Tabla 3.

La Figura 5 indica que para el crecimiento de los alevines de $O$. bicirrhosum, el tratamiento con mayor ganancia de peso fue $\mathrm{T} 1$ para la réplica $\mathrm{R} 4$, con $0,24 \mathrm{~g} /$ día, seguido de R6: 0,23 g/día, y R5: 0,22 g/día. El tratamiento TC presentó ganancias en peso para las réplicas R1: 0,19 g/día, R3: 0,18 g/día y R2: 0,14 g/día. Los alevines con menor ganancia en peso fueron T2 para las réplicas R8: 0,10 g/día y R7: 0,08 g/día, seguidos por R9: 0,07 g/día.

La Figura 6 muestra que para el incremento de talla de los alevines, la mayor ganancia fue $\mathrm{T} 1$ para las réplicas R4, R5 y R6 con un valor de 0,11 $\mathrm{mm} /$ día para las tres replicas. Para el tratamiento con concentrado (TC) se constató que R3 presentó $0,10 \mathrm{~mm} /$ día, seguido de R1 con un valor de $0,9 \mathrm{~mm} /$ día y R2 con $0,8 \mathrm{~mm} /$ día. El tratamiento con menor incremento en la talla fue T2, que en R7 y R8 obtuvieron valores de $0,6 \mathrm{~mm} /$ día y en $\mathrm{R} 9$ : $0,5 \mathrm{~mm} /$ día.
En la Tabla 2 se presentan los valores de crecimiento y talla de las tres dietas alternativas, con un peso inicial de $2,44 \mathrm{~g}$ y una talla de $8,15 \mathrm{~cm}$ para TC, $2,86 \mathrm{~g}$ y $8,25 \mathrm{~cm}$ para T1, y $2,65 \mathrm{~g} \mathrm{y} 8,42 \mathrm{~cm}$ para T2. En un periodo de 78 días de investigación se observó que en el tratamiento $\mathrm{T} 1$ registró un peso final $20,83 \mathrm{~g}$ y una talla de $16,58 \mathrm{~cm}$, con una ganancia de peso por día de $0,230 \mathrm{~g}$ obteniendo mayor ganancia que el tratamiento $\mathrm{TC}$, el cual muestra un peso final de $15,66 \mathrm{~g}$ y una talla de $15,08 \mathrm{~cm}$, con una ganancia de peso diario de $0,169 \mathrm{~g}$. El tratamiento T2 registró el menor peso final $(9,17 \mathrm{~g})$ y la menor talla final $(12,75 \mathrm{~cm})$, con una escasa ganancia de peso diario $(0,084 \mathrm{~g})$ respectivamente.

En la Tabla 3 se exponen los datos de producción. Se muestra las biomasas obtenidas en las tres dietas. con sus respectivas réplicas. Se observa que los individuos sometidos al tratamiento $\mathrm{T} 1$ con la réplica $\mathrm{R} 4$ obtuvieron la mayor ganancia de peso $(18,6 \mathrm{~g})$, seguido de $\mathrm{R} 6$ con 18,2 g. La menor ganancia la obtuvo $\mathrm{T} 2$ con la réplica $\mathrm{R} 9$, registrando un valor de $5,70 \mathrm{~g}$.

Con respecto a la tasa de crecimiento especifico (TCE), el tratamiento que obtuvo el mayor porcentaje fue el $\mathrm{T} 1$ con la réplica $\mathrm{R} 4$ registrando $2,66 \%$, seguido del TC con $2,56 \%$ para $\mathrm{R} 1$ y el menor porcentaje lo obtuvo el tratamiento T2 con la réplica R9 alcanzando un valor de 1,38\%. En cuanto a la TCA, los mayores valores los registró el T1 con la réplica $\mathrm{R} 4$ con un valor de $0,320 \mathrm{~g}$ /día, seguido de R5 con 0,294 g/día, y el menor lo obtuvo T2 con la réplica $R 9$ alcanzando un valor de $0,098 \mathrm{~g} / \mathrm{día}$.

Los datos que se muestran en la Tabla 3 , nos indican una tasa de sobrevivencia del $100 \%$ de los alevines alimentados con el concentrado proteico, para las réplicas R1, R2 y R3, seguido del tratamiento con P. reticulata (guppys) para R4, R5 y R6, y el tratamiento con $C$. formosanus (termitas) para R7 y R8. El valor más bajo correspondió a R9, con el 98\% de sobrevivencia.

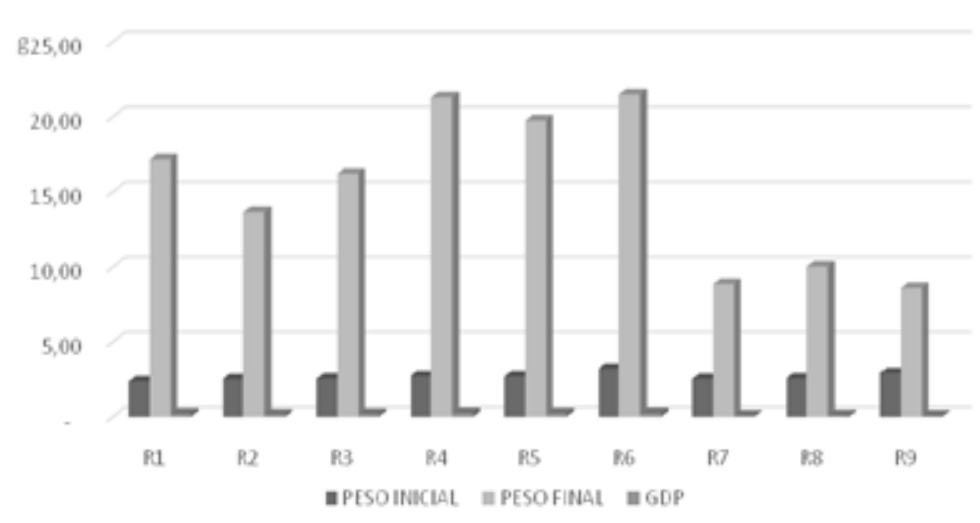

Figura 5. Ganancias de pesos iniciales, finales y diarios (GDP) en los tres tratamientos, con tres replicas cada uno. 


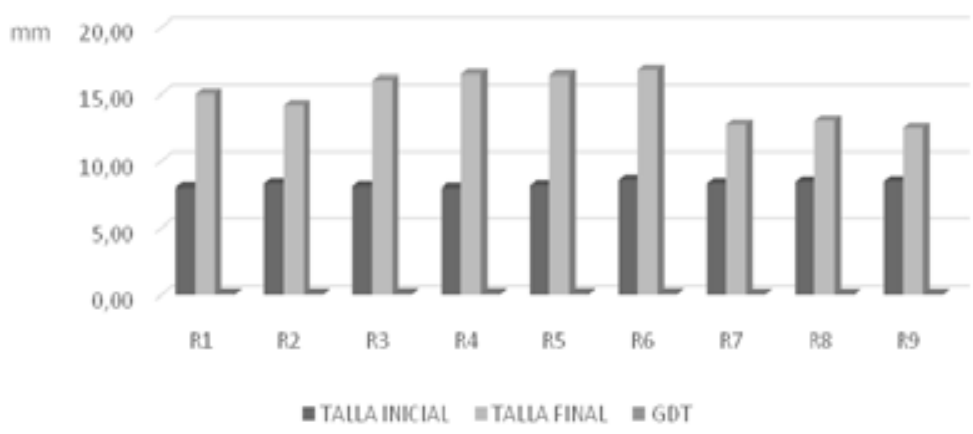

Figura 6. Aumentos de tallas iniciales, finales y diarias (GDT) en los tres tratamientos, con tres réplicas cada uno.

El tratamiento más sobresaliente para esta investigación fue para T1, que obtuvo el mayor incremento de la biomasa total final, con un valor de $21,47 \mathrm{~g} \mathrm{y}$ $21,28 \mathrm{~g}$ en las réplicas R6 y $\mathrm{R} 4$, siendo la réplica $\mathrm{R} 9$ del tratamiento $\mathrm{T} 2$ la que obtuvo menor incremento en la biomasa total final, con 8,62 g. Para el tratamiento con concentrado TC, se obtuvo un incremento de la biomasa total final de $17,13 \mathrm{~g}$, seguido de las réplicas R1 y R3 con 16,18 g y en menor incremento R2 $(13,67$ g). T2 mostro un incremento de biomasa total final de $10,02 \mathrm{~g}, \mathrm{R} 8$ y R7 de $8,87 \mathrm{~g}$, y un menor incremento en $\mathrm{R} 9$ con $8,62 \mathrm{~g}$.

Con respecto a la ganancia de peso, T1 logró el mayor incremento con valores de 18,25 y $18,6 \mathrm{~g}$ en R6 y R4 respectivamente. R5 mostró la menor ganancia de peso $(17,08 \mathrm{~g})$. El tratamiento TC exhibió una ganancia de peso de 14,8 y 13,66 g en R1 y R3, obteniendo menor rendimiento R2, con 11,2 g. El tratamiento T2 obtuvo ganancias de peso de 7,5 y 6,37 g para R8 y R7, asumiento R9 el menor incremento $(5,7 \mathrm{~g})$.

\section{DISCUSIÓN}

Los sistemas de recirculación cerrados han sido históricamente asociados con aplicaciones investigativas dirigidas a la obtención de menores costos productivos $7,18,28$. En el presente trabajo se generan nuevos conocimientos para la producción de $O$. bicirrhosum, habiéndose obtenido resultados de sobrevivencia del $99,7 \%$.

En esta investigación el valor del $\mathrm{pH}$ del agua fue de 6,3 (Figura 3), similar al valor de 6,0 reportado por otros autores ${ }^{15}$. Ello indicó que nuestro $\mathrm{pH}$ fue adecuado para el "levante" de alevines de la especie $O$. bicirrhosum en un SCR. Otros investigadores afirman que los valores de $\mathrm{pH}$ óptimos para la cría de arawanas oscilan entre 6,5 y $8.5^{5}$, superiores a los reportados en esta investigación.

En este trabajo, la calidad del agua a temperatura de $28^{\circ} \mathrm{C}$ fue la apropiada para los diferentes tratamientos (Figura 4). Resultados similares fueron reportados para especies nativas con temperaturas entre $26 \mathrm{y}$ $29^{\circ} \mathrm{C}^{5}$; esto se debe a que la temperatura del territorio andino amazónico presenta registros entre 24 y $28^{\circ} \mathrm{C}^{3}$.
Para las especies $P$. reticulata y C. formosanus, los valores de proteína corporal no pudieron ser hallados en la bibliografía consultada. En esta investigación se analizaron mediante el método Kjeldahl en ambas especies, obteniéndose un $27 \%$ de proteína para los guppys y $22 \%$ para las termitas; es de notar que estas especies contienen nutrientes y energía que influyen en el crecimiento de los alevines. Para $P$. reticulata existen reportes que le asignan porcentajes del $30-40 \%$ de proteína ${ }^{30}$.

Por otra parte, el alimento vivo tiene la cualidad de no afectar la calidad del agua, debido a que es consumido antes de su descomposición, a diferencia del alimento inerte (concentrado peletizado o extrusado), el cual se descompone y afecta al medio, causando mortalidad en el estanque o acuarios, además de retardar el desarrollo de las larvas de los peces, disminuir el crecimiento y reducir la eficiencia de la transformación de energía y materia ${ }^{16}$.

Contrariamente, el alimento vivo tiene cualidades inexistentes en el alimento inerte, como ser el movimiento, que estimula ser atrapado por el depredador; el color, que es atractivo para su captura; la calidad nutritiva, ya que los organismos que se aprovechan como alimento contienen la cantidad y la calidad de nutrimentos indispensables para el adecuado crecimiento de las especies en el agua ${ }^{9}$. Asímismo, el alimento vivo para peces es indispensable durante las primeras semanas para obtener altos porcentajes de sobrevivencia y crecimiento acelerado ${ }^{20}$.

Al evaluar las tres dietas, el tratamiento concentrado con $45 \%$ de proteína obtuvo un peso final de 15,66 g y una talla $15,08 \mathrm{~cm}$, en tanto que el tratamiento con $C$. formosanus reportó un peso $9,17 \mathrm{~g}$ y una talla $12,75 \mathrm{~cm}$. Ambos tratamientos resultaron significativamente inferiores a los obtenidos con la dieta a base de $P$. reticulata (con $27 \%$ de proteína), que originó un peso $20,83 \mathrm{~g}$ y una talla $16,58 \mathrm{~cm}$ (Tabla 2), garantizando la sobrevivencia y el óptimo desarrollo de O. bicirrhosum.

La ganancia de peso final y la longitud final para el tratamiento que obtuvo los mejores resultados ( $\mathrm{T} 1$ : 20,83 g y $16,58 \mathrm{~cm}$ ), resultaron mayores a los obtenidos en otras investigaciones que registraron ganancia de peso final de $11,9 \mathrm{~g}$ y longitud de $15,41 \mathrm{~cm}$ a $\operatorname{los} 70$ días de cultivo de 180 alevines de $O$. bicirrhosum, lapso durante el cual se suplementó la dieta con larvas de díptereos quironómidos y alimento comercial al $50 \%$, con una biomasa del $5 \%$ y $7 \%{ }^{26}$. Tales niveles fueron menores a los utilizados en esta investigación ( $20 \%$ de la biomasa), lo cual mejoró el nivel de crecimiento de los especímenes.

El porcentaje de proteína no necesariamente debe ser superior a los niveles de iniciación (45\%). Prueba de ello es que en su estado silvestre los peces obtienen su 
alimento depredando otras especies, por lo cual la dieta se enriquece con varios nutrientes (grasa, energía) y muestra una mejor asimilación por los peces ${ }^{10}$.

La réplica 9 (Tabla 1) muestra un $98 \%$ de supervivencia de los alevines de arawana, siendo la más baja de todos los tratamientos. El tratamiento con concentrado proteico al $45 \%$ y la dieta con $P$. reticulata, no presentaron mortalidad de individuos, lo cual permite deducir que su contenido de proteínas fue suficiente para mantener la sobrevivencia y el crecimiento.

El sistema de recirculación de agua implementado, así como la calidad y concentración del agua utilizada resultaron apropiadas, permitiendo que las dietas balanceadas con diferentes porcentajes proteicos influyeron positivamente en la sobrevivencia de los alevinos, la cual siempre fue superior al $95 \%{ }^{10}$.

Se concluye que la dieta que presentó mejor rendimiento en cuanto al crecimiento de los alevines de la fase VII de $O$. bicirrhosum fue el alimento vivo con $P$. reticulata ( $27 \%$ de proteínas), con una ganancia de peso de $0,23 \mathrm{~g} /$ día, suministrando un mínimo de $10 \mathrm{~g} /$ día, lo que equivale a un $26,5 \%$ más de aumento de peso que el obtenido con alimento concentrado $(45 \%$ de proteínas). Así, el tratamiento con guppys ( $P$. reticulata) surge como una nueva forma de alimentación, más económica y eficiente para el mantenimiento de alevinos de $O$. bicirrhosum.

\section{REFERENCIAS}

1. Acosta AC, Ortega C, Sanguino WR, Ceballos BL, López JL. 2010. Evaluación de tres tipos de alimento como dieta en post-larvas de sábalo amazónico (Brycon melanopterus, Cope 1872). Vet Zootec 4: 42-50.

2. Alvarado H. 1999. Crecimiento y sobrevivencia de la trucha arco iris cultivada en diferentes tipos de estanques y densidades. Veterinaria Trop 24: 121-129.

3. Arango C, Dorado J, Guzmán D, Ruiz JF. 2012. Cambio climático más probable para Colombia a lo largo del siglo XXI respecto al clima presente. Grupo de modelamiento de tiempo, clima y escenarios de cambio climático, Subdirección de Meteorología, IDEAM, p. 18.

4. Arce E, Luna J. 2003. Efecto de dietas con diferente contenido proteico en las tasas de crecimiento de crías del bagre del balsas Ictalurus balsanus (Pisces: Ictaluridae) en condiciones de cautiverio. Rev Aquatic 18: 39-47.

5. Argumedo EG. 2005. Arawana: manual para la cría comercial en cautiverio, Ed. Produmedios, Bogotá, Colombia, p.105.

6. Bastardo HR, Sofía SB. 2003. Crecimiento de truchas todas hembras y de ambos sexos en un criadero venezolano. Zoot Trop 21: 17-26.

7. Caldwell J. 1998. Why use aquaculture as an educational tool? The Conservation Fund's Freshwater Institute, Shepherdstown, USA, 3: 1-12.

8. Carbó R. 2010. Sistemas de recirculación para la acuicultura SRA. Rev IPAC Acuicultura 47: 16-17.
9. Castro T, Delara A, Castro G, Castro J, Malpica A. 2003. Alimento vivo en la acuicultura. Contactos 48: 2733.

10. Cerna L, Chukoo F, Alcántara F, Mori L. 2014. Efecto de tres dietas comerciales en el crecimiento y sobrevivencia de alevinos de pez ángel, Pterophyllum scalare (Perciformes, Cichildae) variedad marmoleada. Folia Amazonica 23: 79-86.

11. Cruz O. 2003. Efecto de la densidad de cultivo sobre el crecimiento del escalar (Pterophyllum scalare) durante el periodo de levante en acuarios. Tesis de grado, Universidad Nacional de Colombia, Bogotá, p. 50-52.

12. Cuaical TC, Vallejo VE, Franco RH, Sanguino OW. 2013. Efecto de la densidad de siembra y la adición de ácido ascórbico en el cultivo de Osteoglossum bicirrhosum. Revista MVZ Córdoba 18: 3799-3806.

13. Gutiérrez FW, Quispe M, Valenzuela L, Contreras G, Zaldívar J. 2010. Utilización de la proteína dietaria por alevinos de la gamitana, Colossoma macropomum, alimentados con dietas isocalóricas. Rev Peruana Biol 17: 219-223.

14. Hernández C. 2009. Estudio preliminar del levante de juveniles de arawana plateada (Osteoglosuum bicirrhosum) en sistema cerrado de recirculación en la estación experimental Rio Grande en el municipio de Cajica (Cundinamarca). Tesis de Grado, Universidad Militar Nueva Granada, Bogotá, Colombia, p. 15-19.

15. Hernández C, Gómez E, Hurtado H. 2010. Estudio preliminar del levante de juveniles de arawana (Osteoglosuum bicirrhosum) en sistemas cerrados de recirculación. Rev Facult Ciencias, Univ Militar Nueva Granada, Bogotá, 6: 96-113.

16. Kamler E. 1992. Early life history of fish, Ed. Chapman Hall, Londres, p. 211-212.

17. Landines MA, Sanabria AI, Victoria P. 2007. Producción de peces ornamentales en Colombia, Ed. Univ. Nac. Colombia, Bogotá, p. 11-114.

18. Lazur MA, Britt DC. 1997. Pond recirculating production systems. Southern Regional Aquaculture Center, SRAC Publication 455: 1-8.

19. Lechuga A, Tafolla D, García P, Cancino R, Soria R, Manzo S. 2011. Manual de prácticas de laboratorio- $f$ siología animal, Ed. Univ. Michoacana de San Nicolás de Hidalgo, Michoacán, México, p. 28-34.

20. Luna J, Vargas ZT, Figueroa TJ. 2010. Alimento vivo como alternativa en la dieta de larvas y juveniles de Pterophyllum scalare (Lichtenstein, 1823). Avanc Investig Agropec 14: 63-72.

21. Mercado I, García JL, Rosado R, Olaya CW, Segura FF, Brú SB, Tordecilla G. 2006. Cultivo de dorada (Brycon sinuensis Dahl, 1955) en jaulas flotantes a diferentes niveles de proteína. Rev Colomb Cienc Pec 19: 204 211.

22. Mojica JI, Usma JS, Álvarez R, Lasso CA. 2012. Libro rojo de peces dulceacuícolas de Colombia 2012, Ed. Instituto de Investigación de Recursos Biológicos Alexander von Humboldt, Instituto de Ciencias Naturales de la Universidad Nacional de Colombia, Bogotá, p. 66-68. 
23. Montaña CA. 2009. Crecimiento y sobrevivencia en el levante de alevinos de trucha arcoíris (Oncorhynchus mykiss) en sistemas cerrados de recirculación de agua. Tesis de grado, Univ. Milit. Nueva Granada, Colombia, p. 39-40.

24. Poot CA, Salazar RA, Hernández MF. 2009. Evaluación de dietas comerciales sobre el crecimiento de tilapia (Oreochromis niloticus), etapa crianza. $2^{\circ}$ Congr Intern Investig, Chihuahua, México, p. 23-25.

25. Ribeyro O, Guerra F, Rodríguez L, Ismiño R, Núñez J, Chukoo F. 2009. Crecimiento y utilización de alimento en alevinos de arahuana (O. bicirrhosum) alimentados con tres frecuencias alimenticias. Rev Folia Amazónica 18: 7580.

26. Ribeyro O, Ismiño R, Chukoo F, Verdi L, Verastegui M, Castillo JM. 2014. Crecimiento de alevinos de Osteoglossum Bicirrhosum "arahuana plateada" en ambientes controlados influenciados por frecuencias alimenticias. Rev Ciencia Amazónica (Iquitos) 4: 45-53.
27. Rodríguez L. 2012. Efecto de la restricción alimenticia sobre el metabolismo energético y el crecimiento en juveniles de cachama blanca Piaractus brachypomus (Cuvier, 1818). Tesis MSci, Universidad Nacional de Colombia, Bogota, p. 17-20.

28. Timmons MB, Ebeling JM, Piedrahita RH. 2009. Acuicultura en sistemas de recirculación, Ed. Cayuga Aqua Ventures, New York, p. 50-60.

29. Trasviña AG, Cervantes M, Pérez E, Timmons M. 2007. Sistema de recirculación modular para uso familiar/multi-familiar, Ed. Instituto Tecnológico de Boca del Río (ITBoca), Veracruz, México, p. 7-9.

30. Velasco Y, Corredor W. 2011. Requerimientos nutricionales de peces ornamentales de agua dulce: una revisión. Rev MVZ Córdoba 16: 2458-2469. 